\title{
Morphometric Optic Nerve Head Analysis in Glaucoma Patients: A Comparison between the Simultaneous Nonmydriatic Stereoscopic Fundus Camera (Kowa Nonmyd WX3D) and the Heidelberg Scanning Laser Ophthalmoscope (HRT III)
}

\author{
Siegfried Mariacher, ${ }^{1}$ Stephanie Hipp, ${ }^{2}$ Robert Wirthky, ${ }^{3}$ \\ Gunnar Blumenstock, ${ }^{4}$ Karl-Ulrich Bartz-Schmidt, ${ }^{3}$ Focke Ziemssen, ${ }^{3}$ \\ Ulrich Schiefer, ${ }^{3,5,6}$ Bogomil Voykov, ${ }^{3}$ and Kai Januschowski ${ }^{1,3}$ \\ ${ }^{1}$ Knappschaft Eye Clinic, Knappschaft Hospital Saar GmbH, 66280 Sulzbach, Germany \\ ${ }^{2}$ Ophthalmology Clinic, Katharinenhospital, Klinikum Stuttgart, 70174 Stuttgart, Germany \\ ${ }^{3}$ University Eye Clinic Tuebingen, Centre for Ophthalmology, Eberhard Karls University of Tuebingen, 72076 Tuebingen, Germany \\ ${ }^{4}$ Department of Clinical Epidemiology and Applied Biostatistics, Eberhard Karls University of Tuebingen, 72076 Tuebingen, Germany \\ ${ }^{5}$ Institute for Ophthalmological Research, Eberhard Karls University of Tuebingen, 72076 Tuebingen, Germany \\ ${ }^{6}$ Competence Center "Vision Research", Aalen University of Applied Sciences, 73430 Aalen, Germany
}

Correspondence should be addressed to Stephanie Hipp; s.hipp@klinikum-stuttgart.de

Received 11 March 2016; Revised 22 April 2016; Accepted 4 May 2016

Academic Editor: Antonio Queiros

Copyright (C) 2016 Siegfried Mariacher et al. This is an open access article distributed under the Creative Commons Attribution License, which permits unrestricted use, distribution, and reproduction in any medium, provided the original work is properly cited.

\begin{abstract}
Purpose. To investigate the agreement between morphometric optic nerve head parameters assessed with the confocal laser ophthalmoscope HRT III and the stereoscopic fundus camera Kowa nonmyd WX3D retrospectively. Methods. Morphometric optic nerve head parameters of 40 eyes of 40 patients with primary open angle glaucoma were analyzed regarding their vertical cup-todisc-ratio (CDR). Vertical CDR, disc area, cup volume, rim volume, and maximum cup depth were assessed with both devices by one examiner. Mean bias and limits of agreement (95\% CI) were obtained using scatter plots and Bland-Altman analysis. Results. Overall vertical CDR comparison between HRT III and Kowa nonmyd WX3D measurements showed a mean difference (limits of agreement) of $-0.06(-0.36$ to 0.24$)$. For the CDR $<0.5$ group $(n=24)$ mean difference in vertical CDR was $-0.14(-0.34$ to 0.06$)$ and for the CDR $\geq 0.5$ group $(n=16) 0.06(-0.21$ to 0.34$)$. Conclusion. This study showed a good agreement between Kowa nonmyd WX3D and HRT III with regard to widely used optic nerve head parameters in patients with glaucomatous optic neuropathy. However, data from Kowa nonmyd WX3D exhibited the tendency to measure larger CDR values than HRT III in the group with $\mathrm{CDR}<0.5$ group and lower $\mathrm{CDR}$ values in the group with $\mathrm{CDR} \geq 0.5$
\end{abstract}

\section{Introduction}

Glaucoma is one of the leading causes for irreversible blindness in western nations [1]. Morphometric optic nerve head parameters like cup-to-disc-ratio (CDR), cup and rim volume, and mean or maximum cup depth are surrogates for glaucomatous optic neuropathy. These parameters are significantly correlated with visual field indices such as mean deviation (MD) and pattern standard deviation (PSD) $[2,3]$.
While morphometric optic nerve head analysis is useful for detecting early structural changes [4], perimetry is used for monitoring functional changes [5]. The above-mentioned approaches are complementary [6]. Morphometric examinations are an objective way to monitor both manifestation and progression of optic neuropathy by detecting changes in optic nerve head parameters in patients with primary open angle glaucoma and ocular hypertension $[7,8]$. 
Evaluation of the optic nerve head with the historically used two-dimensional planimetry was particularly difficult in patients with extremely small or large discs, regarding detection of glaucomatous damage in small discs and progression in large discs [7]. In 1988 the confocal laser ophthalmoscope Heidelberg Retina Tomograph (HRT) was introduced. A laser diode with a wavelength of $670 \mathrm{~nm}$ was used for scanning purposes $[4,9,10]$. Currently the HRT has become one of the standard tools for three-dimensional topographic analysis of the optic nerve head [11, 12]. The Kowa nonmyd WX3D is a stereometric fundus camera that is able to take two photographs simultaneously $[13,14]$. Thus a highly reproducible (in contrast to sequential shift of a single camera) and most importantly real (in contrast to HRT) stereoscopic photo is acquired and changes can be analyzed qualitatively via anatomic examination as well as quantitatively via stereometric optic nerve head parameters $[15,16]$, per pixel diversion. If the analysis of optic nerve head parameters with Kowa nonmyd WX3D and HRT measurements would be comparable, the virtues of stereoscopic photography and optic nerve head morphometric analysis could be combined. This study was conducted to investigate the agreement between HRT III and Kowa nonmyd WX3D in patients with primary open angle glaucoma.

\section{Materials and Methods}

40 eyes of 40 patients with primary open angle glaucoma were monitored at the glaucoma outpatient ward at the University Eye Hospital Tuebingen and analyzed retrospectively representing a rough average of the possible eligible patient population. Patients with chronic, progressive optic neuropathies with characteristic morphological changes at the optic nerve head and retinal nerve fiber layer in the absence of other ocular disease or congenital anomalies were defined as having an open angle glaucoma. Progressive retinal ganglion cells death and visual field loss are associated with these changes and were mandatory to be included into this analysis. Eligible patients were divided into 2 groups regarding their vertical CDR assessed with HRT III (Heidelberg Retina Tomograph III, Heidelberg Engineering GmbH, Heidelberg, Germany). Patients with a vertical CDR lower than 0.5 formed one group and patients with a vertical CDR higher than or equal to 0.5 formed the second group. Exclusion criteria were media opacities (cornea, lens, and vitreous), advanced ametropia (spherical refraction exceeding 4 diopters or astigmatism exceeding 2 diopters), ophthalmological diseases affecting the optic nerve head other than glaucoma, or impaired quality indices with regard to HRT (namely, topography standard deviation (TSD) > 30 or intertest variation of reference height $>25 \mu \mathrm{m}$ ). Patients who underwent refractive or vitreoretinal surgery were also excluded from the analysis. Corneal pachymetry measurements were included into the analysis for correction of the HRT III results.

The contour line of the disc needs to be placed automatically or manually on the top of the disc margin and serves as a reference for follow-up examinations. The cup contour will be reassessed within each subsequent session [12]. The cup contour line alignment was manually performed in both
Kowa nonmyd WX3D and HRT III by one examiner. Vertical CDR, disc area, cup volume, rim volume, and maximal cup depth were analyzed with both methods. Agreement between HRT III and Kowa nonmyd WX3D (Kowa Company Ltd., Nagoya, Japan) was analyzed using scatter plots and BlandAltman analysis [17]. Mean bias and upper and lower limits of agreement (95\% confidence interval (CI)) were evaluated. All statistical analyses were performed using IBM SPSS Statistics 22.0 for Windows (IBM Corp., Armonk, NY, US).

The current investigation was approved by the ethics committee of the University of Tuebingen (Germany). This study was performed in accordance with the ethical tenets outlined in the Declaration of Helsinki.

\section{Results}

The study included 40 eyes from 40 patients with primary open angle glaucoma. Twenty-four eyes with a CDR $<0.5$ and 16 eyes with a $C D R \geq 0.5$ were analyzed. 27 female patients $(67.5 \%)$ and 13 male patients (32.5\%) were included in this survey. In the group with CDR $<0.518$ female $(75 \%)$ and 6 male $(25 \%)$ patients and in the group with CDR $\geq 0.59$ female (56\%) and 7 male (44\%) patients were investigated.

The agreement between morphometric optic nerve head parameters assessed with HRT III and Kowa nonmyd WX3D was analyzed using mean differences and limits of agreement (95\% CI). Bland-Altman plots and scatter plots were generated to compare and validate measurements from both devices (Figure 1). Table 1 shows the mean CDR, cup volume, rim volume, and maximum cup depth measured with HRT III and Kowa nonmyd WX3D. The mean differences with corresponding limits of agreement between HRT III measurements and Kowa nonmyd WX3D measurements are shown in Table 2.

Overall analysis of vertical CDR showed a slight negative mean difference between HRT III and Kowa nonmyd WX3D measurement ( -0.06 ; limits of agreement -0.36 to 0.24$)$. Mean disc area and rim volume were larger, but cup volume and maximal cup depth were smaller in Kowa nonmyd WX3D assessment in comparison to the HRT III assessment (Table 2).

Scatter plots and Bland-Altman plots (Figure 1) also showed a negative mean difference $(-0.14$; limits of agreement -0.34 to 0.06 ) between vertical CDR assessed with HRT III and Kowa nonmyd WX3D in the CDR $<0.5$ group, indicating that the Kowa nonmyd WX3D was measuring larger CDR values than the HRT III in this group.

Cup volume and maximum cup depth showed a positive mean difference between HRT III and Kowa nonmyd WX3D measurement. Additionally, the disc area and the rim volume are further parameters beside the CDR, displaying a negative mean difference (Table 2).

Comparison of CDR assessment with HRT III and Kowa nonmyd WX3D in the CDR $\geq 0.5$ group showed a positive mean difference (0.06; limits of agreement -0.21 to 0.34 ). However, data from Kowa nonmyd WX3D exhibited the tendency to measure lower CDR values than HRT III in the group with $C D R \geq 0.5$. The same tendency can be seen for the cup volume and the maximum cup depth; only the disc area 


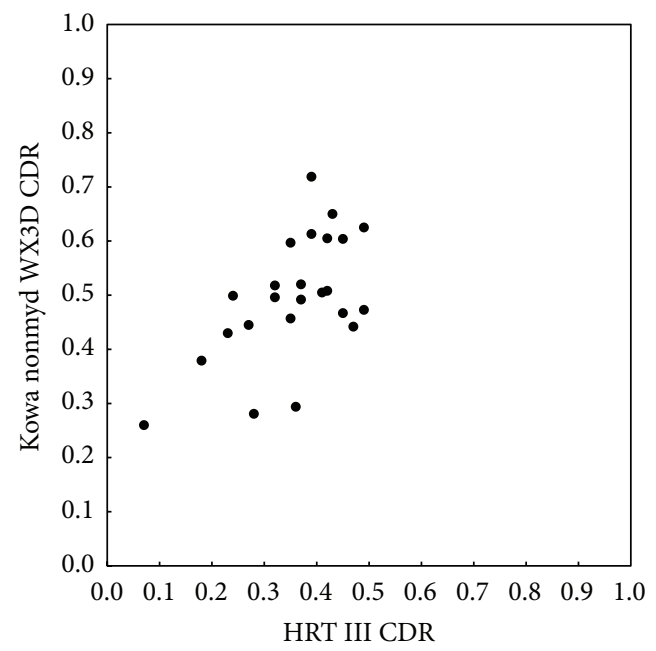

(a)

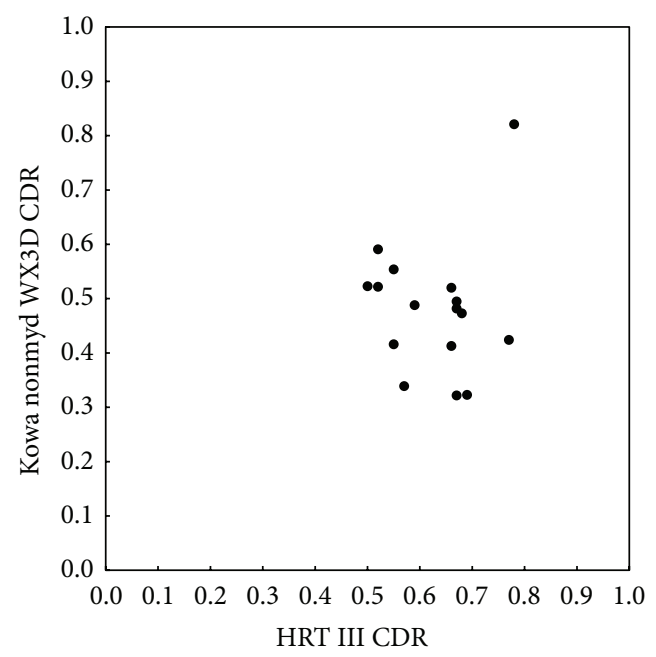

(c)

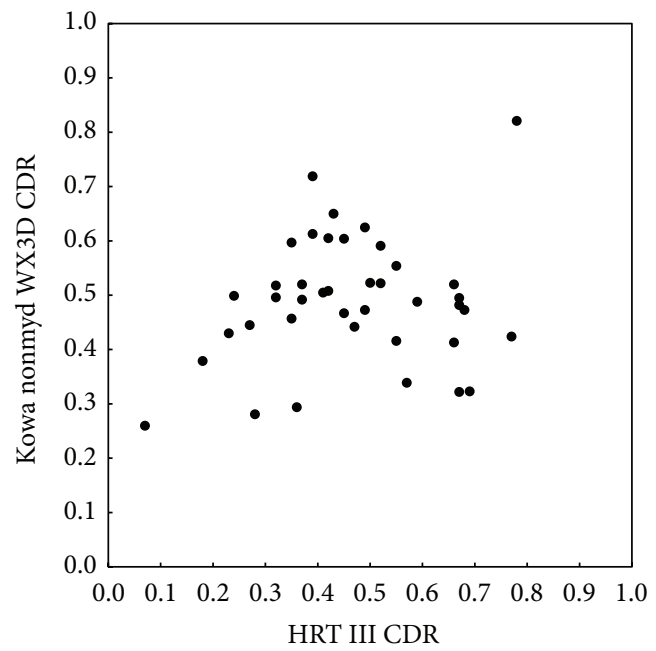

(e)

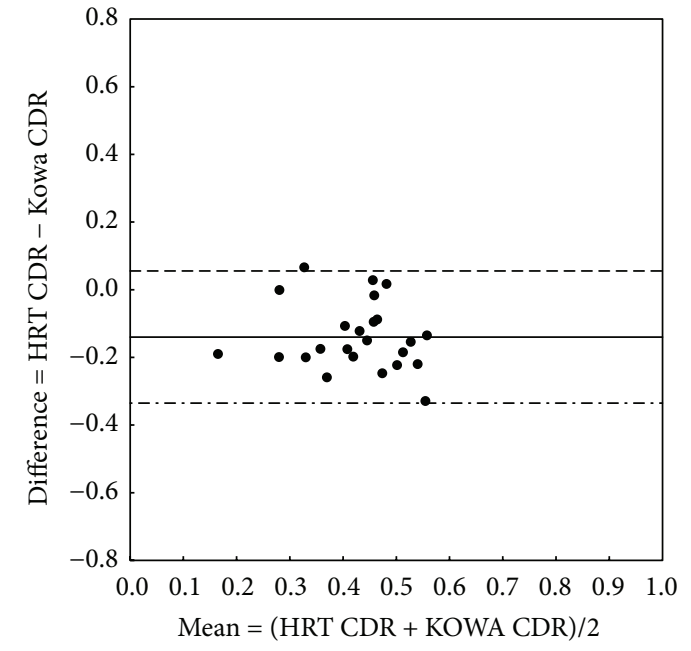

(b)

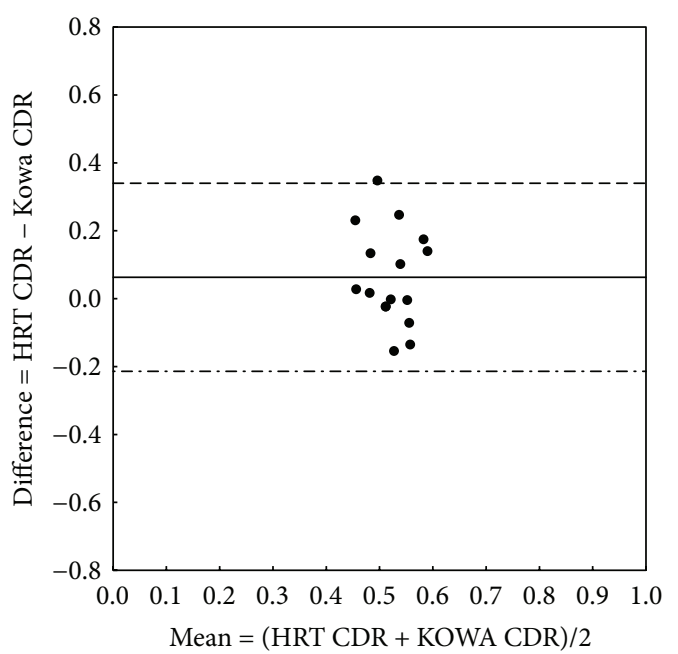

(d)

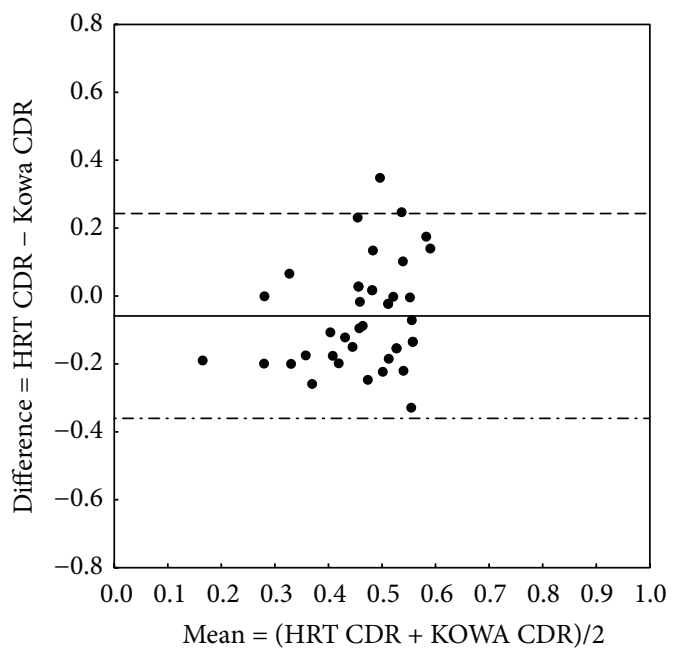

(f)

FIGURE 1: Scatter plots (a, c, e) and Bland-Altman plots (b, d, f) showing the correlation between (vertical) CDR measurements using HRT III versus Kowa nonmyd WX3D, separated for the group with CDR $<0.5$ (a, b) and the group with CDR $\geq 0.5$ (c, d). Overall comparison is visualized in plots (e, f). In Bland-Altman plots solid lines indicate mean differences between CDR measured with HRT III and Kowa nonmyd WX3D. The 95\% limits of agreement are indicated by the upper dashed lines and the lower dot-dashed lines. 
TABLE 1: Comparison and distribution of stereometric optic nerve head parameters assessed with HRT III and Kowa nonmyd WX3D for all investigated patients subdivided in two groups according to their CDR (cut-off value 0.5 ) and in total. Values are expressed as mean ( \pm standard deviation, SD).

\begin{tabular}{|c|c|c|}
\hline $\begin{array}{l}\text { Optic nerve head } \\
\text { parameter }\end{array}$ & $\begin{array}{l}\text { HRT III } \\
(N=24)\end{array}$ & $\begin{array}{c}\text { Kowa nonmyd } \\
\text { WX3D }(N=16)\end{array}$ \\
\hline \multicolumn{3}{|c|}{$\mathrm{CDR}<0.5$ group } \\
\hline CDR & $0.36( \pm 0.10)$ & $0.50( \pm 0.12)$ \\
\hline Disc area $\left(\mathrm{mm}^{2}\right)$ & $2.18( \pm 0.32)$ & $2.83( \pm 0.57)$ \\
\hline Cup volume $\left(\mathrm{mm}^{3}\right)$ & $0.18( \pm 0.10)$ & $0.12( \pm 0.08)$ \\
\hline Rim volume $\left(\mathrm{mm}^{3}\right)$ & $0.38( \pm 0.16)$ & $0.50( \pm 0.35)$ \\
\hline Maximum cup depth (mm) & $0.70( \pm 0.19)$ & $0.38( \pm 0.15)$ \\
\hline \multicolumn{3}{|c|}{ CDR $\geq 0.5$ group } \\
\hline $\mathrm{CDR}$ & $0.63( \pm 0.09)$ & $0.48( \pm 0.12)$ \\
\hline Disc area $\left(\mathrm{mm}^{2}\right)$ & $2.03( \pm 0.45)$ & $2.79( \pm 0.59)$ \\
\hline Cup volume $\left(\mathrm{mm}^{3}\right)$ & $0.30( \pm 0.16)$ & $0.11( \pm 0.08)$ \\
\hline Rim volume $\left(\mathrm{mm}^{3}\right)$ & $0.18( \pm 0.06)$ & $0.51( \pm 0.32)$ \\
\hline Maximum cup depth (mm) & $0.72( \pm 0.25)$ & $0.38( \pm 0.17)$ \\
\hline \multicolumn{3}{|c|}{ Total } \\
\hline CDR & $0.46( \pm 0.17)$ & $0.49( \pm 0.12)$ \\
\hline Disc area $\left(\mathrm{mm}^{2}\right)$ & $2.12( \pm 0.38)$ & $2.82( \pm 0.57)$ \\
\hline Cup volume $\left(\mathrm{mm}^{3}\right)$ & $0.22( \pm 0.14)$ & $0.11( \pm 0.08)$ \\
\hline Rim volume $\left(\mathrm{mm}^{3}\right)$ & $0.30( \pm 0.16)$ & $0.50( \pm 0.33)$ \\
\hline Maximum cup depth (mm) & $0.70( \pm 0.21)$ & $0.38( \pm 0.15)$ \\
\hline
\end{tabular}

and the rim volume showed a negative mean difference in the CDR $\geq 0.5$ group (Table 2 ).

\section{Discussion}

This study evaluated the agreement of optic nerve head parameters obtained by Kowa nonmyd WX3D and HRT III with regard to the vertical CDR in glaucomatous patients for the first time. Comparison of optic nerve head parameters assessed with HRT III and Kowa nonmyd WX3D suggests a good agreement between those two devices indicated by the results of the Bland-Altman plots. Analysis showed the tendency to overestimate the CDR with Kowa nonmyd WX3D in relation to the HRT III measurements in the CDR $<0.5$ group. CDR values measured with the Kowa nonmyd WX3D in the CDR $\geq 0.5$ group were lower than HRT III CDR values, indicating the tendency to underestimate CDR with Kowa nonmyd WX3D in comparison to HRT III values in this group. These results are supported by earlier studies [1820].

HRT III is an established tool for imaging and quantifying morphometric optic nerve head parameters. Optic nerve head assessment tools like OCT and GdX analysis do not include 3-dimensional optic disc evaluation. Furthermore, GdX data are of limited agreement with the HRT and OCT measurements can differ significantly between various OCT devices $[21,22]$. In contrast to the HRT III, GdX, and OCT, the stereoscopic fundus images obtained by the Kowa nonmyd WX3D camera allow an additional evaluation of the optic disc regarding important in vivo parameters such as pallor, bleeding, and general appearance and can therefore serve as an additional assessment tool for optic nerve head vitality.

Optic nerve head imaging and especially HRT III have gained widespread use in monitoring optic nerve head parameters in patients with glaucoma or suspected glaucoma $[23,24]$. Optic nerve head parameters assessed with HRT were demonstrated to be reproducible and help detect the onset and monitoring progression of glaucomatous optic neuropathy [10]. Test-retest variability has been extensively studied in the recent past and depends on patient age, severity of glaucoma, image quality, cylindrical error, lens opacity, surface geometry, and reference plane: in good-quality HRT images, the sensitivity for detecting $80 \%$ of rim area change of $-0.012 \mathrm{~mm}^{2}$ per year requires, however, 4 examinations per year, which may not be feasible in everyday practice. Reducing the number of examinations to 2 per year reduces the sensitivity to $60 \%$ with simultaneous $20-30 \%$ false positive rate [25]. On the other hand stereographic photography is an accepted tool for optic disc assessment in glaucoma patients $[26,27]$. Sensitivity and specificity for detecting progression of stereometric optic nerve head parameters with optic nerve head photography were analyzed in previous studies and seem to be limited: the best correlating optic nerve head parameter was the CDR $[19,28]$. Reference height differences and the mean topography standard deviation (TSD) indicated image quality and were therefore influencing sensitivity and specificity of the optic nerve head assessment [29]. Further factors affecting the sensitivity and specificity including lens opacification and astigmatism have been found as factors affecting the measurement variability of this kind of optic disc tomography $[28,30,31]$ and were therefore valuated as exclusion criteria.

The test-retest repeatability has been shown to improve with increasing CDR values $\left(R^{2}=0.21 ; p<0.01\right)$ in HRT measurements [32]. In this study test-retest repeatability was not investigated or compared. As only glaucoma patients were included, one could assume a rather large than a small CDR. However, our analyses divided the study population into two groups in accordance with the vertical CDR with a cut-off value of CDR 0.5 . Thus differentiation between severity levels is solely based on a CDR classification and therefore limited. Furthermore functional aspects (like visual field parameters) are not included in this survey, so results from this study refer exclusively to morphometric but not functional criteria.

In this retrospective study both methods used a manual contour line alignment, which is a major source of measurement variability, even though simultaneous stereoscopic viewing of the optic nerve head photographs is known to facilitate the drawing of the contour line [24]. The contour line from the baseline measurement is normally automatically transferred to the follow-up measurement, which is also a common feature to reduce variability of CDR value comparison between a baseline and a follow-up measurement. The manually defined contour line affects the comparison of optic nerve head parameters in this study, due to visualized differences in optic disc parameters between 
TABLE 2: Mean differences [limits of agreement (95\% CI)] of stereometric optic nerve head parameters between HRT III and Kowa nonmyd WX3D assessment separated for the CDR $<0.5$ group and the CDR $\geq 0.5$ group and in total.

\begin{tabular}{lccc}
\hline Optic nerve head parameter & CDR $<0.5(N=24)$ & CDR $\geq 0.5(N=16)$ & Total $(N=40)$ \\
\hline CDR & $-0.14[-0.34$ to 0.06$]$ & $0.06[-0.21$ to 0.34$]$ & $-0.06[-0.36$ to 0.24$]$ \\
Disc area $\left(\mathrm{mm}^{2}\right)$ & $-0.66[-1.73$ to 0.42$]$ & $-0.76[-1.92$ to 0.40$]$ & $-0.70[-1.80$ to 0.40$]$ \\
Cup volume $\left(\mathrm{mm}^{3}\right)$ & $0.06[-0.11$ to 0.23$]$ & $0.19[-0.07$ to 0.46$]$ & $0.11[-0.13$ to 0.36$]$ \\
Rim volume $\left(\mathrm{mm}^{3}\right)$ & $-0.12[-0.87$ to 0.63$]$ & $-0.32[-0.95$ to 0.28$]$ & $-0.20[-0.93$ to 0.53$]$ \\
Maximum cup depth $(\mathrm{mm})$ & $0.32[-0.04$ to 0.68$]$ & $0.34[-0.01$ to 0.69$]$ & $0.33[-0.02$ to 0.68$]$ \\
\hline
\end{tabular}

HRT III and Kowa nonmyd WX3D (Figure 1), even though the mentioned difference in the disc area as a surrogate for the manually drawn contour line is within a small range between the two groups (Table 2). Recent HRT III software releases offer an automated analysis mode for optic nerve head assessment, which does not require prior manual outlining of a contour line [33]. Automated contour line drawing is still not commonly used, so manually selected outlining of disc boundaries was chosen in this study despite the abovementioned variability. Nevertheless one should be cautious when interpreting transferred values between HRT III and Kowa nonmyd WX3D.

\section{Conclusion}

In conclusion, Kowa nonmyd WX3D is a potential tool for monitoring optic nerve head parameters quantitatively and qualitatively, not only as an adjunct or alternative to the HRT. Principle limitations of the study are the manual contour line alignment, the retrospective nature, and the very small sample sizes. However, Kowa nonmyd WX3D exhibited the tendency to measure larger CDR values than HRT III in the group with $\mathrm{CDR}<0.5$ and lower $\mathrm{CDR}$ values in the group with $\mathrm{CDR} \geq 0.5$.

\section{Competing Interests}

The authors have no competing interests to declare.

\section{References}

[1] R. R. A. Bourne, J. B. Jonas, S. R. Flaxman et al., "Prevalence and causes of vision loss in high-income countries and in Eastern and Central Europe: 1990-2010," British Journal of Ophthalmology, vol. 98, no. 5, pp. 629-638, 2014.

[2] E. M. Hoffmann, S. Miglior, T. Zeyen et al., "The Heidelberg retina tomograph ancillary study to the European glaucoma prevention study: study design and baseline factors," Acta Ophthalmologica, vol. 91, no. 8, pp. e612-e619, 2013.

[3] U. Schiefer, J. Pätzold, B. Wabbels, and F. Dannheim, "Conventional techniques of visual field examination: part 4: static perimetry: interpretation-perimetric indices-followup-perimetry in childhood," Ophthalmologe, vol. 103, no. 3, pp. 235-256, 2006.

[4] J. B. Jonas, N. X. Nguyen, and G. O. H. Naumann, "Optic disc morphometry in simple optic nerve atrophy," Acta Ophthalmologica, vol. 67, no. 2, pp. 199-203, 1989.
[5] J. W. Cho, K. R. Sung, S.-C. Yun, J. H. Na, Y. Lee, and M. S. Kook, "Progression detection in different stages of glaucoma: mean deviation versus visual field index," Japanese Journal of Ophthalmology, vol. 56, no. 2, pp. 128-133, 2012.

[6] A. Sommer, J. Katz, H. A. Quigley et al., "Clinically detectable nerve fiber atrophy precedes the onset of glaucomatous field loss," Archives of Ophthalmology, vol. 109, no. 1, pp. 77-83, 1991.

[7] K. U. Bartz-Schmidt, M. Sündtgen, R. A. Widder, J. Weber, and G. K. Krieglstein, "Limits of two-dimensional planimetry in the follow-up of glaucomatous optic discs," Graefe's Archive for Clinical and Experimental Ophthalmology, vol. 233, no. 5, pp. 284-290, 1995.

[8] M. A. Kass, D. K. Heuer, E. J. Higginbotham et al., "The Ocular Hypertension Treatment Study: a randomized trial determines that topical ocular hypotensive medication delays or prevents the onset of primary open-angle glaucoma," Archives of Ophthalmology, vol. 120, no. 6, pp. 701-713, 2002.

[9] K. U. Bartz-Schmidt, J. Weber, and K. Heimann, "Validity of two-dimensional data obtained with the Heidelberg Retina Tomograph as verified by direct measurements in normal optic nerve heads," German Journal of Ophthalmology, vol. 3, no. 6, pp. 400-405, 1994.

[10] K. Rohrschneider, R. O. W. Burk, F. E. Kruse, and H. E. Völcker, "Reproducibility of the optic nerve head topography with a new laser tomographic scanning device," Ophthalmology, vol. 101, no. 6, pp. 1044-1049, 1994.

[11] R. N. Weinreb, "Assessment of optic disc topography for diagnosing and monitoring glaucoma," Archives of Ophthalmology, vol. 116, no. 9, pp. 1229-1231, 1998.

[12] L. Kalaboukhova, V. Fridhammar, and B. Lindblom, "Glaucoma follow-up by the Heidelberg retina tomograph-new graphical analysis of optic disc topography changes," Graefe's Archive for Clinical and Experimental Ophthalmology, vol. 244, no. 6, pp. 654-662, 2006.

[13] M. Iester, F. S. Mikelberg, P. Courtright et al., "Interobserver variability of optic disk variables measured by confocal scanning laser tomography," American Journal of Ophthalmology, vol. 132, no. 1, pp. 57-62, 2001.

[14] Y. Yokoyama, M. Tanito, K. Nitta et al., "Stereoscopic analysis of optic nerve head parameters in primary open angle glaucoma: the glaucoma stereo analysis study," PLoS ONE, vol. 9, no. 6, Article ID e99138, 2014.

[15] T. Nakagawa, T. Suzuki, Y. Hayashi et al., "Quantitative depth analysis of optic nerve head using stereo retinal fundus image pair," Journal of Biomedical Optics, vol. 13, no. 6, Article ID 064026, 2008. 
[16] M. M. Iester, G. Wollstein, R. A. Bilonick et al., "Agreement among graders on Heidelberg retina tomograph (HRT) topographic change analysis (TCA) glaucoma progression interpretation," British Journal of Ophthalmology, vol. 99, no. 4, pp. 519523, 2015.

[17] J. M. Bland and D. G. Altman, "Statistical methods for assessing agreement between two methods of clinical measurement," The Lancet, vol. 1, no. 8476, pp. 307-310, 1986.

[18] K. Januschowski, G. Blumenstock, C. E. Rayford II, K.-U. BartzSchmidt, U. Schiefer, and F. Ziemssen, "Stereometric parameters of the optic disc. Comparison between a simultaneous non-mydriatic stereoscopic fundus camera (KOWA WX 3D) and the Heidelberg scanning laser ophthalmoscope (HRT IIII)," Ophthalmologe, vol. 108, no. 10, pp. 957-962, 2011.

[19] R. O. Burk, K. Rohrschneider, T. Takamoto, H. E. Völcker, and B. Schwartz, "Laser scanning tomography and stereophotogrammetry in three-dimensional optic disc analysis," Graefe's Archive for Clinical and Experimental Ophthalmology, vol. 231, no. 4, pp. 193-198, 1993.

[20] F. S. Mikelberg, G. R. Douglas, M. Schulzer, P. J. Airaksinen, K. Wijsman, and D. Mawson, "The correlation between cup-disk ratio, neuroretinal rim area, and optic disk area measured by the video-ophthalmograph (Rodenstock analyzer) and clinical measurement," American Journal of Ophthalmology, vol. 101, no. 1, pp. 7-12, 1986.

[21] C. K.-S. Leung, C. Y.-L. Cheung, R. N. Weinreb et al., "Retinal nerve fiber layer imaging with spectral-domain optical coherence tomography: a variability and diagnostic performance study," Ophthalmology, vol. 116, no. 7, pp. 1257-1263.e2, 2009.

[22] C. K.-S. Leung, V. Chiu, R. N. Weinreb et al., "Evaluation of retinal nerve fiber layer progression in glaucoma: a comparison between spectral-domain and time-domain optical coherence tomography," Ophthalmology, vol. 118, no. 8, pp. 1558-1562, 2011.

[23] C. Breusegem, S. Fieuws, I. Stalmans, and T. Zeyen, "Variability of the standard reference height and its influence on the stereometric parameters of the Heidelberg Retina Tomograph 3," Investigative Ophthalmology and Visual Science, vol. 49, no. 11, pp. 4881-4885, 2008.

[24] P. Sharma, P. A. Sample, L. M. Zangwill, and J. S. Schuman, "Diagnostic tools for glaucoma detection and management," Survey of Ophthalmology, vol. 53, no. 6, supplement, pp. S17S32, 2008.

[25] M. Araie, "Test-retest variability in structural parameters measured with glaucoma imaging devices," Japanese Journal of Ophthalmology, vol. 57, no. 1, pp. 1-24, 2013.

[26] M. J. Greaney, D. C. Hoffman, D. F. Garway-Heath, M. Nakla, A. L. Coleman, and J. Caprioli, "Comparison of optic nerve imaging methods to distinguish normal eyes from those with glaucoma," Investigative Ophthalmology and Visual Science, vol. 43, no. 1, pp. 140-145, 2002.

[27] R. A. Stone, G.-S. Ying, D. J. Pearson et al., "Utility of digital stereo images for optic disc evaluation," Investigative Ophthalmology and Visual Science, vol. 51, no. 11, pp. 5667-5674, 2010.

[28] V. Saarela, A. Falck, P. J. Airaksinen, and A. Tuulonen, “The sensitivity and specificity of Heidelberg Retina Tomograph parameters to glaucomatous progression in disc photographs," British Journal of Ophthalmology, vol. 94, no. 1, pp. 68-73, 2010.

[29] V. Saarela, A. Falck, P. J. Airaksinen, and A. Tuulonen, "Factors affecting the sensitivity and specificity of the Heidelberg Retina Tomograph parameters to glaucomatous progression in disc photographs," Acta Ophthalmologica, vol. 90, no. 2, pp. 132-138, 2012.
[30] N. G. Strouthidis, E. T. White, V. M. F. Owen, T. A. Ho, C. J. Hammond, and D. F. Garway-Heath, "Factors affecting the test-retest variability of Heidelberg retina tomograph and Heidelberg retina tomograph II measurements," British Journal of Ophthalmology, vol. 89, no. 11, pp. 1427-1432, 2005.

[31] R. Sihota, V. Gulati, H. C. Agarwal, R. Saxena, A. Sharma, and R. M. Pandey, "Variables affecting test-retest variability of Heidelberg Retina Tomograph II stereometric parameters," Journal of Glaucoma, vol. 11, no. 4, pp. 321-328, 2002.

[32] T. S. Prata, C. G. V. De Moraes, C. C. Teng, C. Tello, R. Ritch, and J. M. Liebmann, "Factors affecting rates of visual field progression in glaucoma patients with optic disc hemorrhage," Ophthalmology, vol. 117, no. 1, pp. 24-29, 2010.

[33] N. V. Swindale, G. Stjepanovic, A. Chin, and F. S. Mikelberg, "Automated analysis of normal and glaucomatous optic nerve head topography images," Investigative Ophthalmology and Visual Science, vol. 41, no. 7, pp. 1730-1742, 2000. 


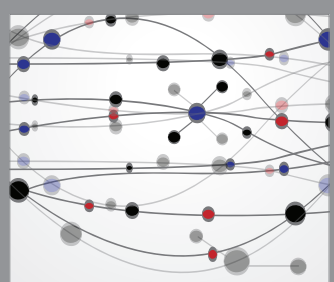

The Scientific World Journal
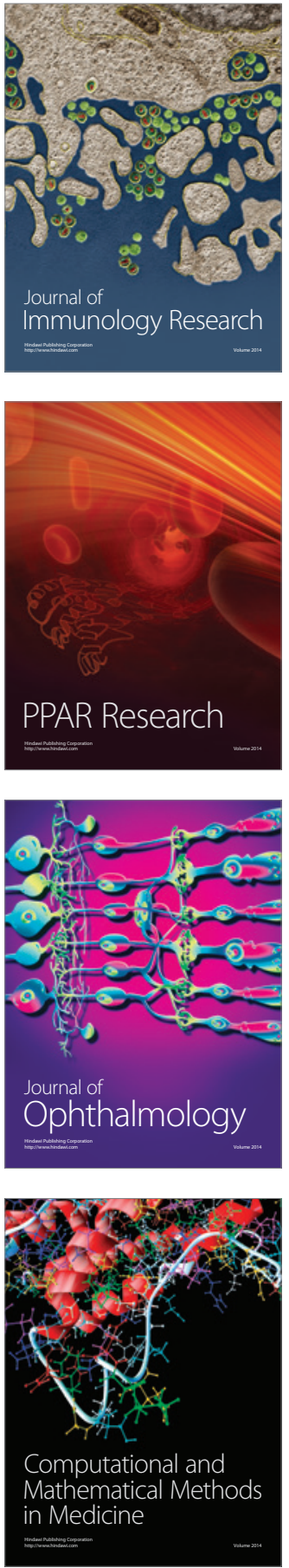

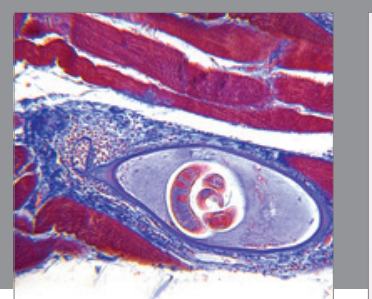

Gastroenterology Research and Practice

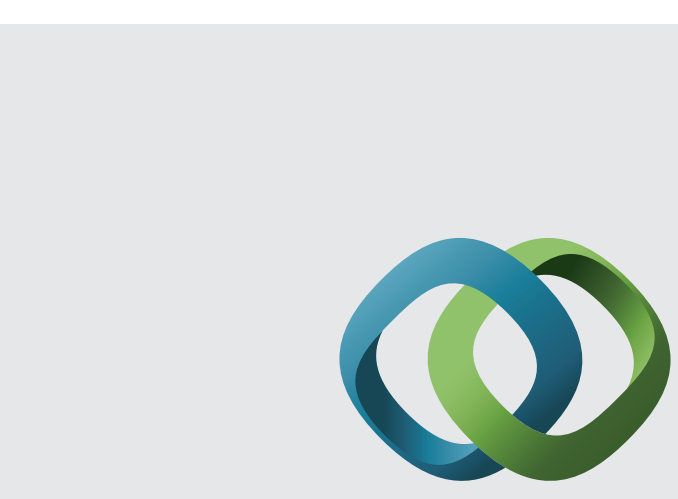

\section{Hindawi}

Submit your manuscripts at

http://www.hindawi.com
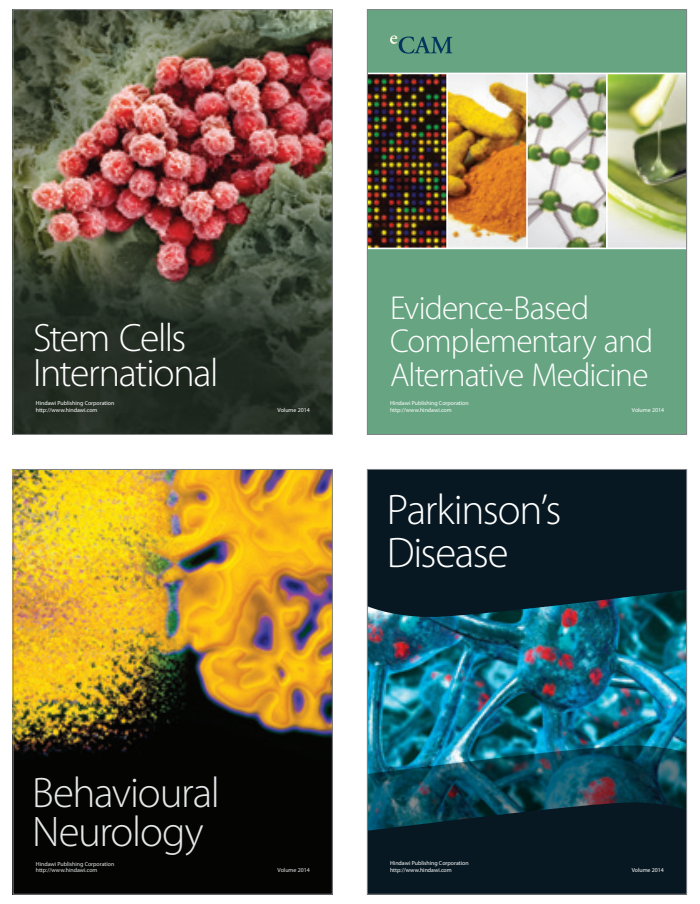
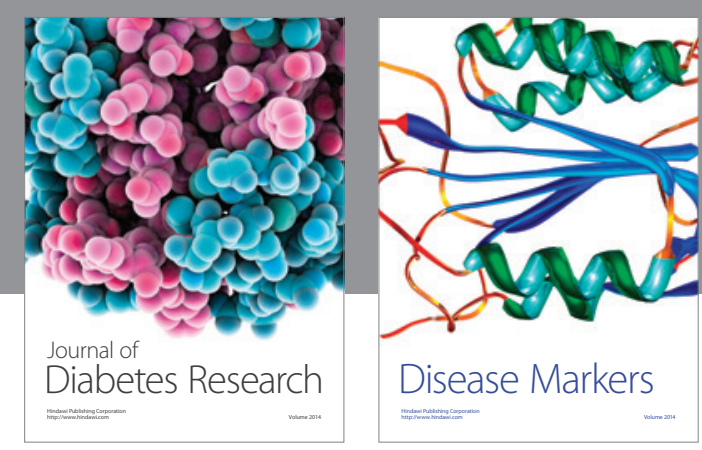

Disease Markers
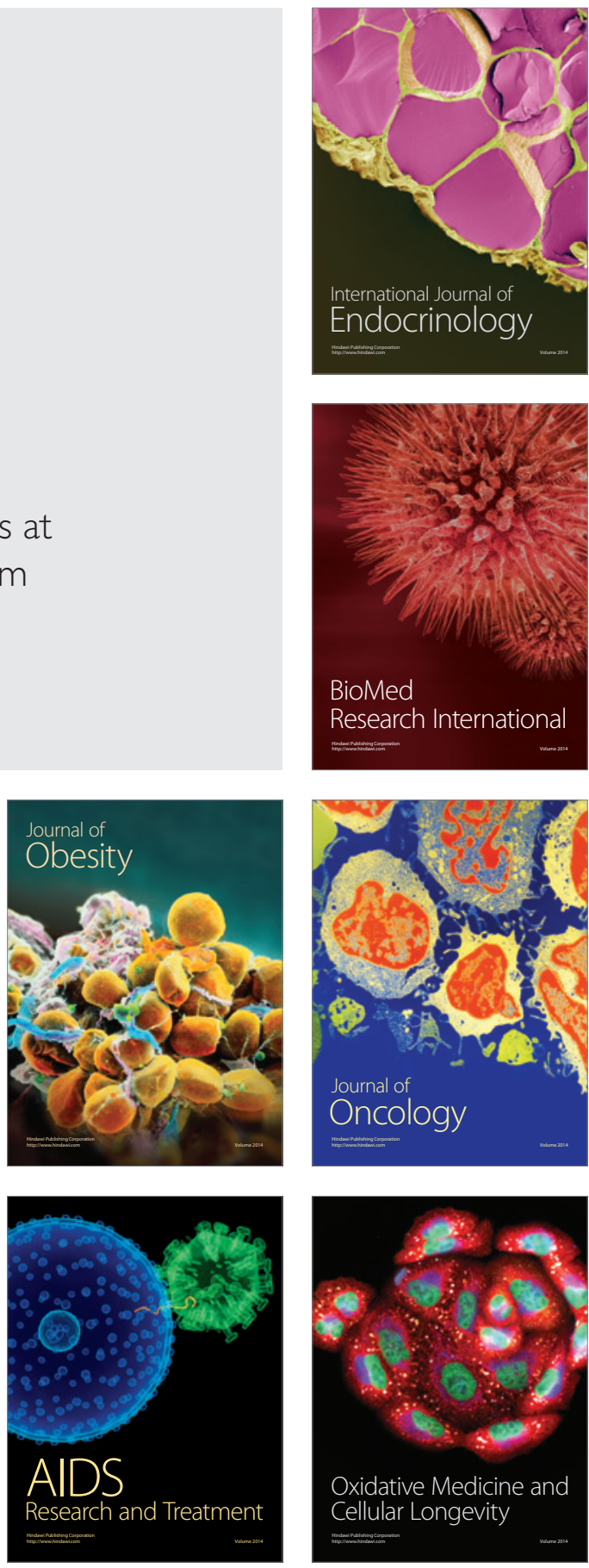